\title{
"The Modern Urological Rehabilitation for Men. Erectile Disorders and Urinary Incontinence. How to Make a Reha-Training to be More Interesting and More Effective. Project Andros - The New Therapeutic Project"
}

\author{
(Erectile Dysfunction, Penile Rehabilitation and Urinary Incontinence after Prostatectomy) \\ Andrzej Przybyla, MD, PhD \\ Urologist, Andrologist \\ CEO Medical Continence Centers \& Mens Health Institute in Warsaw, Poland. \\ doctor@reha-urology.com; andrzej.przybyla@centrumkontynencji.pl \\ medicalcontinencecenters.eu
}

*Corresponding Author: Andrzej Przybyla, MD, PhD, Urologist, Andrologist CEO Medical Continence Centers \& Mens Health Institute in Warsaw, Poland.

\section{Abstract}

Introduction: It was reported in literature that $>80 \%$ of men suffer from urinary incontinence after radical prostatectomy. The symptoms can be treated conservativ (pelvic floor training) or by surgery. The conservative therapy with supervised urological rehabilitation/rehabilitative urology is a standard and an effective and attractive method for men with urinary incontinence after radical prostatectomy. Rehabilitative Urology / Urological rehabilitation in men after radical prostatectomy results in a higher effectivity and patient's acceptance.

Material and Methods: Own pictures and materials.

Conclusions: Erection disorder and urinary incontinence are the basic symptoms of patients who had radical prostate surgeries. In many countries of Western Europe, this surgery is not equivalent with the end of treatment(!). The patient, in order to get closer to the functional state he was before the surgery, has to undergo an intensive rehabilitation under the supervision of urologists and physiotherapists in a specialized clinic. Urology rehabilitation / Rehabilitative Urology is a distinct and very important element of the further treatment! This branch of rehabilitation focuses on urologic diseases and treatment of post operative complications, such as previously mentioned erectile dysfunction or urinary incontinence.

Health-promoting actions and projects for men should be more interesting and specifically oriented to the "stronger sex". The modernity and effectiveness along with an encouraging and (particularly for men) interesting form of the program is necessary in both the prevention and the treatment therapies. This trend is noticeable not only in hospital medicine but also in rehabilitation.

The most common urological illnesses among men are erectile dysfunction and urinary incontinence. They can have organic or physical causes or they can result from the old age.

On a daily basis, I run a modern clinic of Urological Rehabilitation \& Aging Male in Germany. I am an urology specialist and since many years I have dealt with the topic of Aging Male, erectile dysfunction and incontinence, which are the main problems of my Patients. 
“The Modern Urological Rehabilitation for Men. Erectile Disorders and Urinary Incontinence. How to Make a Reha-Training to be More Interesting and More Effective. Project Andros - The New Therapeutic Project"

During my daily work I noticed that the men after the radical prostatectomy or radiotherapy, who suffer from erectile dysfunction and/or urinary incontinence, are not entirely satisfied with the "normal", classical training of the pelvic floor muscles.

The prompt for the new training idea was the observation that for many men the "normal" training of the continence is unattractive (considered as a "female gymnastics"). This "masculine" approach to the subject caused the training of the continence/ potency to be more attractive by the use of, among other things, electromiographically (EMG) tested exercises that were transferred directly onto the training equipment.

The effect of this observation was the introduction of the new elements to the therapy, which are not only effective but also attractive for men. This is how the Project Andros was born.

The Project Andros is the latest solution in the conservative therapy of the erectile dysfunction and/

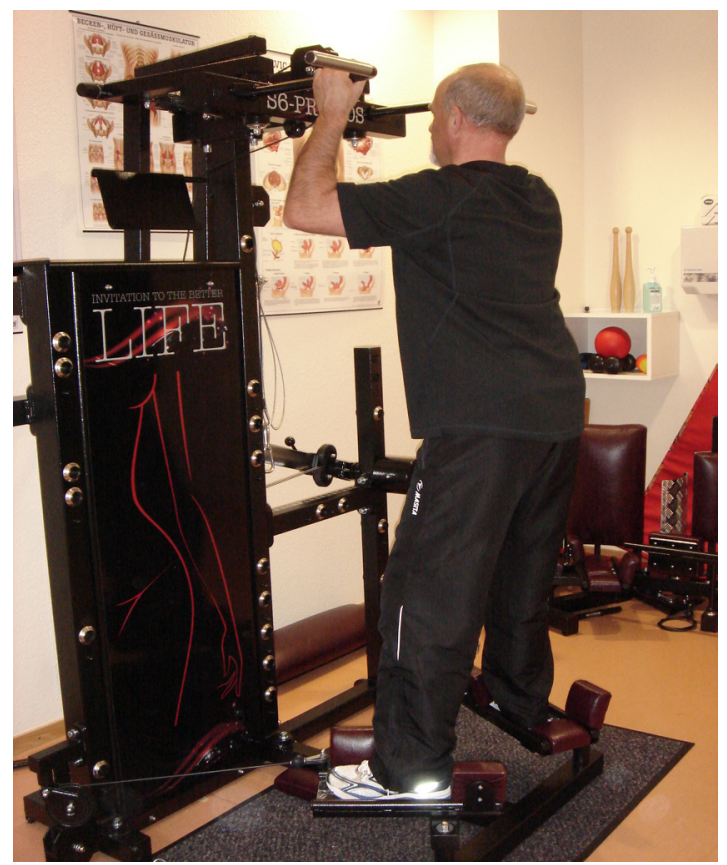

Example 1

Patient age 63, 2 weeks after the radical prostatectomy (da Vinci, nerve sparing), BMI 28

Urinary incontinence before the admission to the clinic (24h Pad-Test in accordance with ICS): Day/night: $271 \mathrm{ml} / 51 \mathrm{ml}$ or urinary incontinence for the patients after the prostatectomy. The training is performed on a special equipment and can be done alone or as a complement to the classical exercises. Particularly noteworthy is the high attractiveness of this form of therapy in comparison to the "regular", classical training performed without any equipment.

During the Training with the Equipment the Achieved Contraction of the Pelvic Floor Muscles and Erectile Muscles are Up to 3-4 Times Higher than in the Classical Training

The training of potency and continence performed on the equipment is done for 5 days per week $(3 \mathrm{x}$ Core Training $+2 x$ Interval Training) during the whole inpatient care period (3-5 weeks). The training should be then continued on an outpatient basis.

Here some of the examples, which show what can be achieved by a patient during such training performed for 3-4 weeks after the surgery.

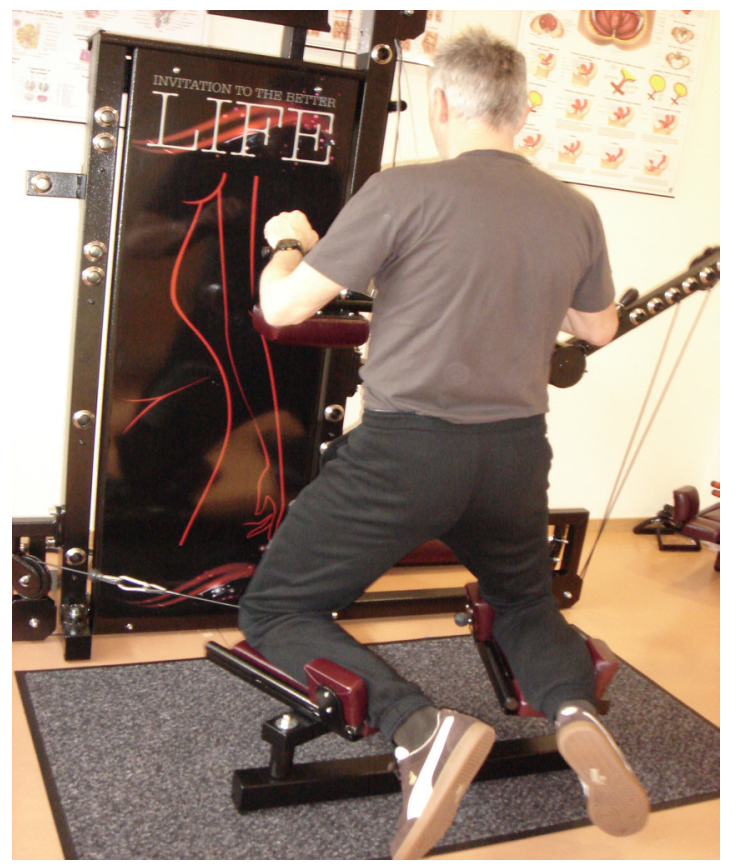

The incontinence measured after 3 weeks of regular exercises

Day/night: $13 \mathrm{ml} / 9 \mathrm{ml}$

\section{Example 2}

Patient age 62, 10 days after the radical prostatectomy (da Vinci, w/o nerve sparing), BMI 30 
"The Modern Urological Rehabilitation for Men. Erectile Disorders and Urinary Incontinence. How to Make a Reha-Training to be More Interesting and More Effective. Project Andros - The New Therapeutic Project"

Urinary incontinence before the admission to the Results after 4 weeks: pain scale reduced to 0-2 clinic (24h Pad-Test in accordance with ICS):

Day/night: $552 \mathrm{ml} / 227 \mathrm{ml}$

Example 5

The incontinence measured after 3 weeks of regular exercises

Day/night: $333 \mathrm{ml} / 128 \mathrm{ml}$

\section{Example3}

Patient age 60,12 days after the radical prostatectomy (da Vinci, nerve sparing), BMI 23

Urinary incontinence before the admission to the clinic (24h Pad-Test in accordance with ICS):

Day/night: $89 \mathrm{ml} / 76 \mathrm{ml}$

The incontinence measured after 4 weeks of regular exercises

Day/night: $17 \mathrm{ml} / 17 \mathrm{ml}$

\section{Example 4}

Patient age 58,

Diagnosis: chronic pelvic pain after the chronic prostatitis and TUR-P (transurethral resection of the prostate).

Pain scale from1 to 10: 7-8 (opioid drugs)

Duration of the therapy: 4 weeks of the Project Andros training

Patient age 56, 2 weeks after the radical prostatectomy (da Vinci, nerve sparing), BMI 26

Urinary incontinence before the admission to the clinic (24h Pad-Test in accordance with ICS):

"almost dry" - minimal incontinence

The incontinence measured after 4 weeks of regular exercises:

Complete continence (100\%)

Potency before the surgery: normal (IEEF>22)

Potency after the surgery, before the admission to the clinic: (IEEF=13) - "lack of morning and nocturnal erections"

Potency measured after 4 weeks of the training: (IEEF=18) - "noticeable return of night and morning erections"

\section{Comment}

The research in the topic of erectile dysfunction is still in progress. The first results of the Patients with the erectile dysfunction after the prostatectomy are very encouraging not only among the Patients, who use the Project Andros only but also for the Patients who participate in the 'mixed' program (Project Andros + tadalafil $5 \mathrm{mg}$ ).

Citation: Andrzej Przybyla, MD, PhD. The Modern Urological Rehabilitation for Men. Erectile Disorders and Urinary Incontinence. How to Make a Reha-Training to be More Interesting and More Effective. Project Andros - The New Therapeutic Project. Archives of Urology. 2019; 2(1): 1-3.

Copyright: (C) 2019 Andrzej Przybyla, MD, PhD. This is an open access article distributed under the Creative Commons Attribution License, which permits unrestricted use, distribution, and reproduction in any medium, provided the original work is properly cited. 